\title{
Fine-Tuning the Electrostatic Properties of an Alkali-Linked Organic Adlayer on a Metal Substrate
}

\author{
Andrea Floris, ${ }^{\dagger, *}$ Alessio Comisso, ${ }^{\dagger}$ and Alessandro De Vita ${ }^{t, *}$ \\ ${ }^{\dagger}$ Department of Physics, King's College London, London, Strand WC2R 2LS, United Kingdom and ${ }^{\ddagger}$ CENMAT, Via Valerio 2, 34127 Trieste, Italy
}

\begin{abstract}

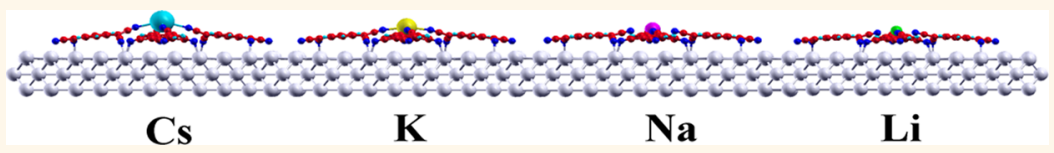

The performance of modern organic electronic devices is often determined by the electronic level alignment at a metal-organic interface. This property can be controlled by introducing an interfacial electrostatic dipole via the insertion of a stable interlayer between the metallic and the organic phases. Here, we use density functional theory to investigate the electrostatic properties of an assembled structure formed by alkali metals coadsorbed with 7,7,8,8-tetracyanoquinodimethane (TCNQ) molecules on a $\mathrm{Ag}(100)$ substrate. We find that the interfacial dipole buildup is regulated by the interplay of adsorption energetics, steric constraints and charge transfer effects, so that choosing chemical substitutions within TCNQ and different alkali metals provides a rich playground to control the systems' electrostatics and in particular fine-tune its work-function shift.
\end{abstract}

KEYWORDS: self-assembly $\cdot$ organic electronics $\cdot$ DFT $\cdot$ level alignment $\cdot$ interfacial dipole $\cdot \mathrm{TCNQ} \cdot$ alkali

$\mathrm{n}$ recent years there has been an increasing interest in using molecular films for the production of organic electronic devices. Thin films made of small molecules or organic polymers can have very interesting semiconducting properties, which can be exploited for the production of OLEDs, OFETs and solar cells.

While these technologies are still the object of very intensive research, many products are now commercially available and fairly widespread. Typically, the electronic properties of the interface between the metal electrodes and the organic semiconductor strongly affect the efficiency of these devices. In particular, there is interest in improving the level alignment across the metal-organic (MO) interface, which is crucial to reduce the carrier injection barriers, in turn strongly affected by the presence of interfacial dipoles. Much effort ${ }^{1}$ has thus been put toward the theoretical understanding of the key processes and features that contribute to the buildup of these dipoles, such as charge transfers, chemical bonding, pillow effect, intrinsic dipoles and metal-induced gap states in the organic semiconductor. ${ }^{2,3}$
Despite this progress, our prediction capabilities are still limited, ${ }^{4}$ and the device design is often a trial and error process. Several attempts have been made to improve the level alignment across $\mathrm{MO}$ interfaces. A common approach is to dope the organic semiconductor in order to shift its HOMO/LUMO levels, for example, using molecular electron acceptors as dopants to create charge transfer complexes., ${ }^{5,6}$ Alkali metals are also exploited as dopants, thanks to their low ionization potential and their strong donor properties. ${ }^{7}$ Alternative procedures are based on the insertion of additional layers (interlayers) between the metallic electrode and the organic semiconductor, which modify the interface dipole. ${ }^{8-11}$ For example, thin interlayers of organic molecules $^{12}$ and molecular acceptors can positively modify the work-function and the injection barriers of noble metal substrates.

The various methods used for tuning the work function, and in particular the insertion of interlayers, have recently resulted in the successful production of prototype devices with improved efficiency, ${ }^{13-16}$ which is promising from a manufacturing point of view. * Address correspondence to
andrea.floris@kcl.ac.uk.

Received for review June 27, 2013 and accepted August 22, 2013.

Published online August 22, 2013 $10.1021 / \mathrm{nn} 403274$ s

() 2013 American Chemical Society 
This work is based on the idea that this could eventually be achieved by inserting a self-assembled monolayer, i.e, a structure that is self-fabricated upon deposition, and could also modify the metal electrode's work-function and the charge carrier injection barrier, e.g., in an organic field effect transistor. ${ }^{17}$ Recently, charge transfer complexes have been used in this context, ${ }^{18,19}$ showing interesting effects on hole- injection barriers. More generally, self-assembly can lead to the formation of a wide range of twodimensional structures with different mechanical and electrostatic properties ${ }^{20-22}$ and appears to be a very promising route for the nanofabrication of interlayers. Our starting point is the recently observed selfassembled monolayer containing Cs alkali metals and electronegative organic 7,7,8,8-tetracyanoquinodimethane (TCNQ) molecules, ${ }^{23}$ which has been shown to assemble in large, compact, and stable chiral domains on $\mathrm{Ag}(100)$ substrates.

In this work we investigate the possibility of extending the use of $\mathrm{Cs}$ to other alkali metals $(\mathrm{Li}, \mathrm{Na}, \mathrm{K}$ ) to tune the electrostatic properties of MO interfaces incorporating these monolayers, with the ultimate goal of extending both the range and the tunability of the interfacial dipoles and electrode work-function. We also suggest the possibility to achieve further control of these properties by evaporating on the substrate mixtures of alkali metals and molecular derivatives with increased electronic affinities. In this respect, we analyze the effects of replacing some TCNQ hydrogen atoms with fluorine atoms and find that this simple substitution produces sizable effects on the electrostatic properties, without affecting the assembly stability.

\section{RESULTS AND DISCUSSION}

Observed Chiral Superstructure: The $\mathrm{Cs}_{-} \mathrm{TCNQ}_{4}$ Network. A self-assembled organic salt formed by $\mathrm{Cs}$ alkali atoms coadsorbed with TCNQ molecules has been recently observed to form very stable and extended domains on the $A g(100)$ substrate, even at room temperature. Cs and TCNQ form intermediate windmill Cs-TCNQ 4 complexes (Figure 1a), which aggregate in strictly chiral domains, forming a commensurate $(6,3,-3,6)$ square superstructure (Figure $1 b, c$ ). The domain size can be very large-typically more than $300 \times 300 \mathrm{~nm}^{2}$-and much larger than the one obtained by a similar network realized with $\mathrm{Mn}$ transition metal. This is the result of an assembly where the adlayer/substrate and the intercomplex interactions in combination with local features of the Cs-TCNQ bond are suitable to make the adlayer particularly stable.

Specific properties influence the physics and the stability of the system: the large electron affinity of TCNQ $(A=4.23 \mathrm{eV})^{24}$ makes it acquire charge both from the surface and from $C s$, which fully donates its valence electron and establishes ionic, nondirectional $\mathrm{Cs}-\mathrm{N}$ bonds, with an associated high rotational freedom of the molecules around the metal. This large flexibility makes the complexes particularly adaptable to different steric constraints and allows maximizing the number of $\mathrm{N}-\mathrm{Ag}$ bonds (three per molecule, Figure 1c) that drive the surface-adlayer interaction energetics, while preserving the hydrogen bonds mediating the intercomplex attractive interaction. The charge acquired by TCNQ makes its central ring aromatic, further enhancing the molecular flexibility ${ }^{25}$ and the ability of the complexes to adapt within the network. Importantly, the nitrogen atoms move toward the substrate upon adsorption/assembly, while the $\mathrm{Cs}^{+}$cations are liftedup with respect to the four $\mathrm{N}$ ligand plane, resulting in a highly nonplanar umbrella structure (Figure 2). The $\mathrm{Cs}^{+}$-topped structure is associated with a positive local electric dipole, which screens the surrounding negative dipoles associated with the molecules and achieves further structural stability through electrostatic screening. Clearly, varying the alkali ion height changes the interfacial dipole. We thus propose that this mechanism could be exploited to tune the electrostatic properties of the interface, based on the hypothesis that different alkali ions will relax at different heights, while preserving the stability of the superstructure described above.

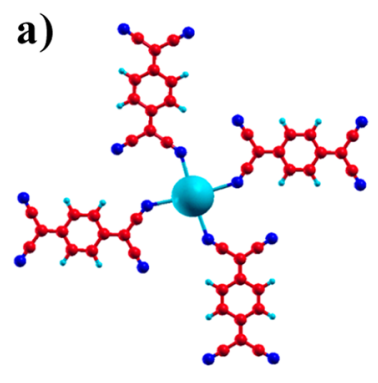

b)

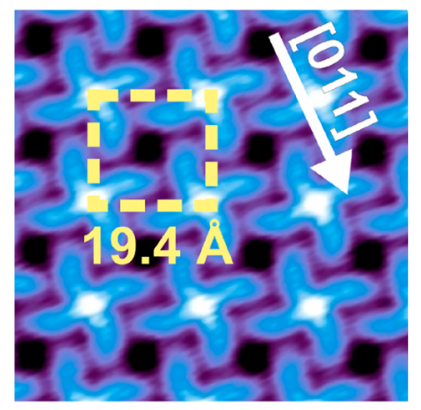

c)

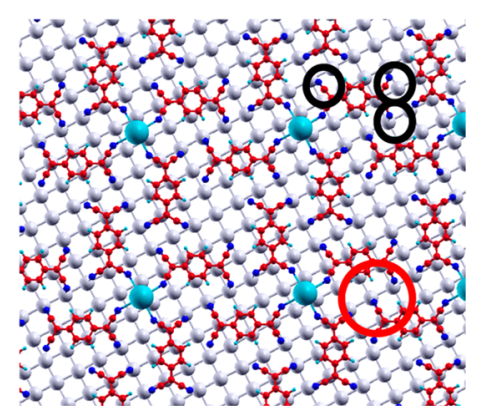

Figure 1. (a) Cs-TCNQ 4 windmill complex structure; (b) STM image showing the chiral self-assembly observed on the $\mathrm{Ag}(100)$ substrate (courtesy of N. Abdurakhmanova); (c) DFT relaxed $(6,3,-3,6)$ square network, with one complex per unit cell. Cs atoms are in light blue (Cs-Cs distance is $19.4 \AA$ ). In (c), the black circles highlight the three $\mathrm{N}-\mathrm{Ag}$ bonds/TCNQ, while the red circle highlights the two hydrogen-bonds/TCNQ. $\mathrm{N}-\mathrm{Ag}$ and the hydrogen bonds are the key features driving the monolayer self-assembly. 


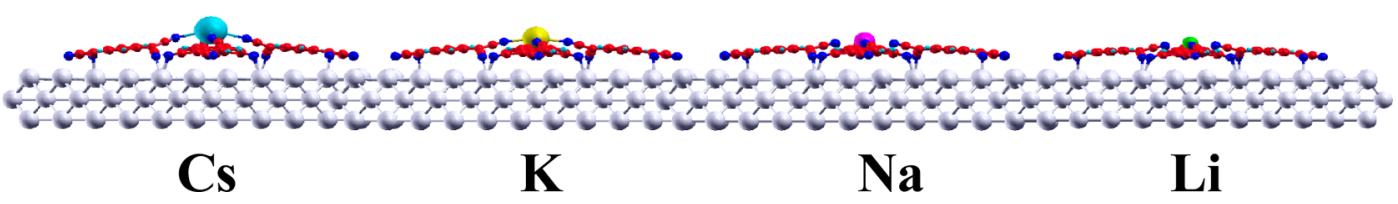

Figure 2. Highly nonplanar umbrella structure achieved by the windmill complexes as a function of the alkali metal center.

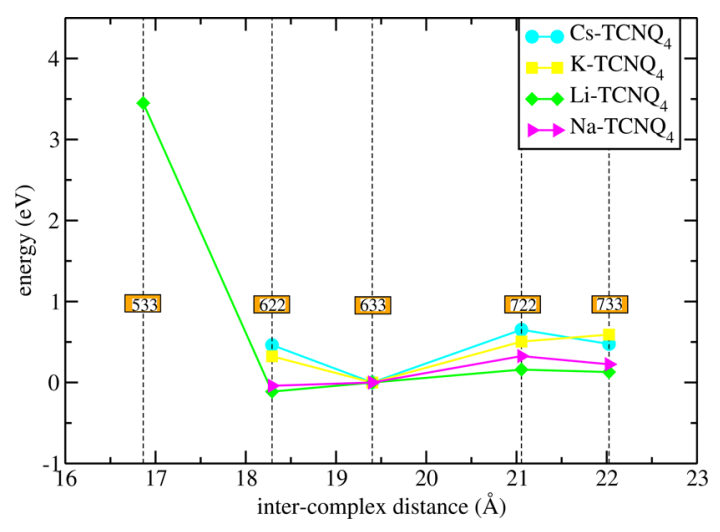

Figure 3. Total energy of periodically repeated networks in the gas phase at different intercomplex distances. The energy zero is set at the $(6,3,-3,6)$ configuration (denoted here 633) for all alkali-TCNQ 4 . We predict that the heavier alkali $(\mathrm{K}, \mathrm{Cs})$ are stable in 633 , while light alkali $(\mathrm{Li}, \mathrm{Na})$ slightly prefer 622 . The latter configuration, however, is unstable on $\mathrm{Ag}(100)$ (see the $\mathrm{SI})$. At lower intercomplex distances the energy increase is due to steric effects, while at larger distances, to the hydrogen bonds breaking. To model the substrate effects, during the relaxation we constrained the nitrogens not bonded with the metal to a specific $z$-coordinate, while the complexes were free to relax in a nonplanar configuration.

TCNQ Coadsorbed with Lighter Alkali: Li, Na, and K. All these alkali atoms have a low ionization potential and are expected to form TCNQ windmill complexes stabilized by ionic bonds upon adsorption on $\mathrm{Ag}(100)$, exactly as observed in the $\mathrm{Cs}$ case, and on $\mathrm{Au}(111)$ for $\mathrm{Li}$ and $\mathrm{Na}^{26}$ However, they have different ionic radii, which increase going from Li to Cs (see Figure 4c). ${ }^{27}$

For each alkali metal $M$, we relax the periodically repeated gas phase $\mathrm{M}-\mathrm{TCNQ}_{4}$ complex network for several intercomplex distances using periodic boundary conditions [see also Supporting Information (SI). Similar calculations of $\mathrm{Mn}$ - and $\mathrm{Cs}_{-}-\mathrm{TCNQ}_{4}$ networks were able to recognize the experimental configuration as the energy minimum]. Through this analysis we clearly identify two superstructures, $(6,2,-2,6)$ and $(6,3,-3,6)$, as the most probable for all the alkali metals (Figure 3). While the lighter $\mathrm{Li}$ and Na might prefer the more compact assembly $(6,2,-2,6)$, K prefers $(6,3,-3,6)$, like the experimentally observed Cs. We then relax these two superstructures on a $\mathrm{Ag}(100)$ substrate, focusing on the lighter $\mathrm{Li}$ and $\mathrm{Na}$. Interestingly, in the more compact superstructure, we find that the windmill complexes become unstable (see the SI). While a full phase space exploration is not viable and we cannot thus rule out the possibility that much more complicated arrangements may form, we interpret the $(6,2,-2,6)$ instability as a preference for the $(6,3,-3,6)$ superstructure even for the lighter alkali.

All systems studied in the $(6,3,-3,6)$ superstructure exhibit a highly nonplanar "umbrella-like" structure (Figure 2). Even lithium, small enough to sit in the plane formed by the $\mathrm{N}$ ligands, relaxes by raising its $z$-coordinate, indicating a residual tendency to move further away from the substrate to increase the positive local dipole. Crucially, the different final height of the alkali ions strongly influences the dipoles and workfunction shifts, as reported in Figure 4. As we move from Cs to Li, we observe a decrease of the umbrella effect and the related intrinsic positive dipole $\left(D_{\text {adl }}\right)$ associated with the M-linked (overall neutral) organic layer.

The moderate increase of the induced dipole $D_{\text {ind }}$ can be attributed only to differences in the adlayer geometries, as further calculations including the metal substrate indicate that the substrate-to-adlayer charge transfer (CT) is the same for all systems ( $\sim 0.37 \mathrm{e} / \mathrm{TCNQ}$ ) (see the $\mathrm{SI}$ ). Importantly, while the $D_{\text {adl }}$ and $D_{\text {ind }}$ dipoles partially compensate each other, they do this to a different degree depending on the alkali. This results in an increase of the predicted work-function shift going from Cs to Li. The predicted shifts are within a $0.46-0.67 \mathrm{eV}$ range. These results clearly lead to the conclusion that a more continuous tuning within this range could be easily achieved by evaporating alkali mixtures, so that the self-assembled monolayer contains complexes with different metal centers.

TCNQ Fluorinated Derivatives. Another promising route for fine-tuning the electrostatic properties of the structures is provided by introducing chemical substitutions within the TCNQ unit molecules forming our assembly complexes. Here we consider fluorinated derivatives (FD-TCNQ), where some or all the hydrogen atoms are substituted with fluorine atoms. This further increases the molecular electronic affinity and can be used to shift the interface dipole toward more negative values, increasing the work-function positive shift. These substitutions also allow us to further explore the relation between charge transfer processes and screening effects achieved by the metal ion position.

Several FD-TCNQ are commercially available, the affinity increasing with the level of fluorination. ${ }^{24}$ First we consider 2,3,5,6-tetrafluoro-7,7,8,8-tetracyanoquinodimethane (F4-TCNQ), where all $\mathrm{H}$ atoms are substituted with $\mathrm{F}$ atoms. This molecule has $A=5.08 \mathrm{eV}^{24}$ ( $0.85 \mathrm{eV}$ higher than TCNQ), and its ability to increase the work-function of several substrates has been 

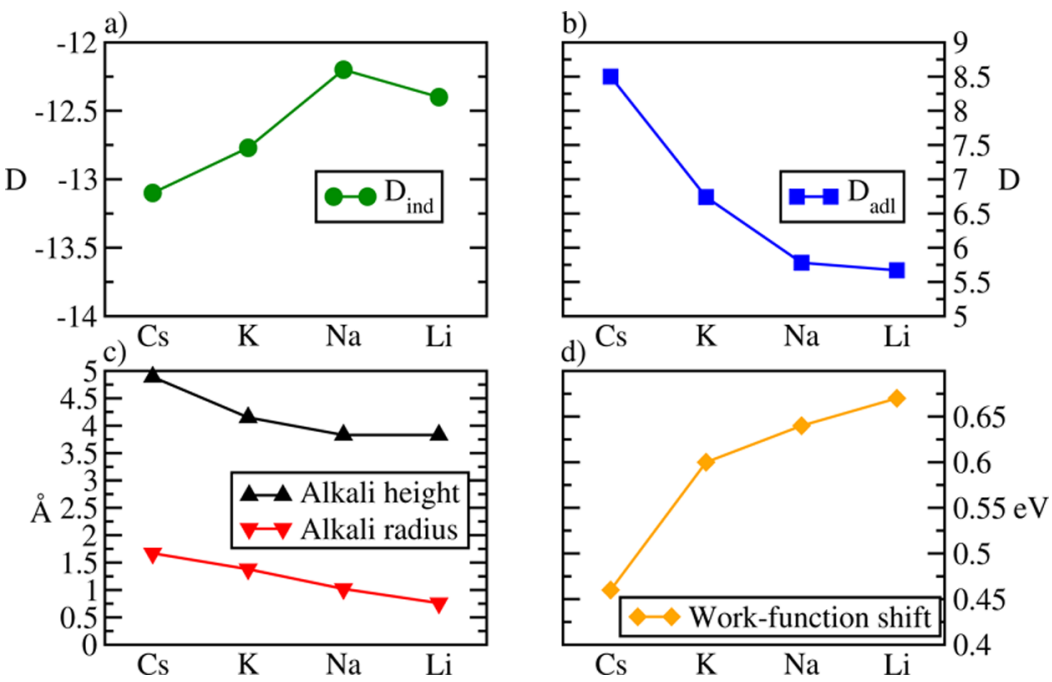

Figure 4. Alkali-dependent properties for alkali- $\mathrm{TCNQ}_{4}$ complexes on $\mathrm{Ag}(100)$. (a) Induced dipole $D_{\text {ind, }}$, due to the substrate/ adlayer charge transfer. The $D_{\text {ind }}$ increase is realized because the same transferred charge is distributed at a lower quota (from Cs to $\mathrm{Li}$ ). (b) Intrinsic dipole $D_{\text {adl, }}$ defined as the dipole of the electrically neutral adlayer in its adsorbed geometry. Its value correlates with the decreasing alkali quota. (c) Alkali ionic radii ${ }^{27}$ (red) and quota (black) in its adsorbed geometry with respect to the substrate. (d) Plot of the work-function shift, showing a clear trend of increase as we move toward lighter alkali. The shift is proportional to the total dipole $D_{\text {ind }}+D_{\text {adl }}$ and is calculated as in ref 37.

already reported. ${ }^{28,29}$ When coadsorbed with molecular donors, it reduces the hole-injection barrier between Au electrodes and several organic semiconductors. ${ }^{30,31}$ We then consider the doubly substituted 2,5-difluoro7,7,8,8-tetracyanoquinodimethane (F2-TCNQ, $A=4.59 \mathrm{eV}$ ) and fluoro-7,7,8,8-tetracyanoquinodimethane (F-TCNQ, $A=4.55 \mathrm{eV}$ ), where only one $\mathrm{H}$ is replaced (see the $\mathrm{SI}$ for the molecular structures on the substrate).

As in the case of non-F-substituted molecules, we first analyze the stability of FD-TCNQ ${ }_{4}$ complexes in gas phase, adsorbed with one of the alkali metals, Cs. In this case we find that the Cs-(F4-TCNQ) ${ }_{4}$ complexes do not show any appreciable level of binding (see Figure SI-3), since the intercomplex hydrogen bonds are completely absent. This indicates that a complete substitution of $\mathrm{H}$ with $\mathrm{F}$ would probably lead to an assembly completely different from the chiral superstructure investigated here (while small quantities of F4-TCNQ as substitutional dopant might still presumably not alter this superstructure and still influence its electrostatic properties). Our calculations indicate that the F-TCNQ and F2-TCNQ derivatives, on the other hand, form the same number of hydrogen bonds as TCNQ in the self-assembled network. We considered two alternative configurations for the $\mathrm{Cs}-(\mathrm{F}-\mathrm{TCNQ})_{4}$ complexes, which can both recreate the same $\mathrm{H}$-bonding pattern. In one case the fluorine atom is on the side of the molecule where one $\mathrm{N}$ is involved in the ionic bond (F1s in Figure 5). In the second case the fluorine is on the opposite side (F10). For both these structures our calculations once more predict a stable $(6,3,-3,6)$ superstructure, which we relax on the substrate (see Figure SI-2). A Cs-(F4-TCNQ) 4 model system was, finally, also relaxed on a $\mathrm{Ag}(100)$ substrate, to establish lower and upper limits for the induced dipole and the Cs screening dipole, respectively, when F4-TCNQ is used as a dopant.

The electrostatic properties at the interface containing FD-TCNQ are presented in Figure 5. As a general consideration, the presence of fluorine produces a clear $D_{\text {ind }}$ decrease, due to the higher electron affinity $A$ and the correspondingly larger charge transfer. The most spectacular dipole decrease (as much as $\sim 9 \mathrm{D}$ ) is associated with the fully substituted derivative F4, with an almost doubled work-function shift as compared with nonsubstituted Cs-linked TCNQ complexes, labeled $\mathrm{T}$ in Figure 5. These values provide an estimate for the energy range where the electrostatic tuning is possible using these dopants. The $D_{\text {ind }}$ decrease is clearly less pronounced when the level of fluorination decreases. In all these structures the Cs atom is pushed up considerably (if compared to the nonfluorinated complexes) and its height once more clearly correlates with the degree of fluorination (cf. Figure $5 b$ ). We find that this effect is partially due to the repulsion between $\mathrm{F}$ and $\mathrm{N}$ ligands, which locally push the alkali up via the local ionic bond, but also to the more subtle, already mentioned long-range effect whereby the increased dipole associated with the positive alkali atom screens the stronger molecular negative dipole. Both effects bring electrostatic stabilization, as confirmed by the $D_{\text {adl }}$ values, in all cases higher than in the reference T system. (See also the SI. Notably, $D_{\text {adl }}$ for F1s is lower than F1o even if the Cs occupies a higher position. This is due to the negative contribution to the dipole of the fluorine, which also stands higher.)

In summary, our calculations predict that introducing TCNQ fluorinated derivatives does not destabilize the adlayer since the flexibility of the complexes can compensate for the additional charge transfer by 

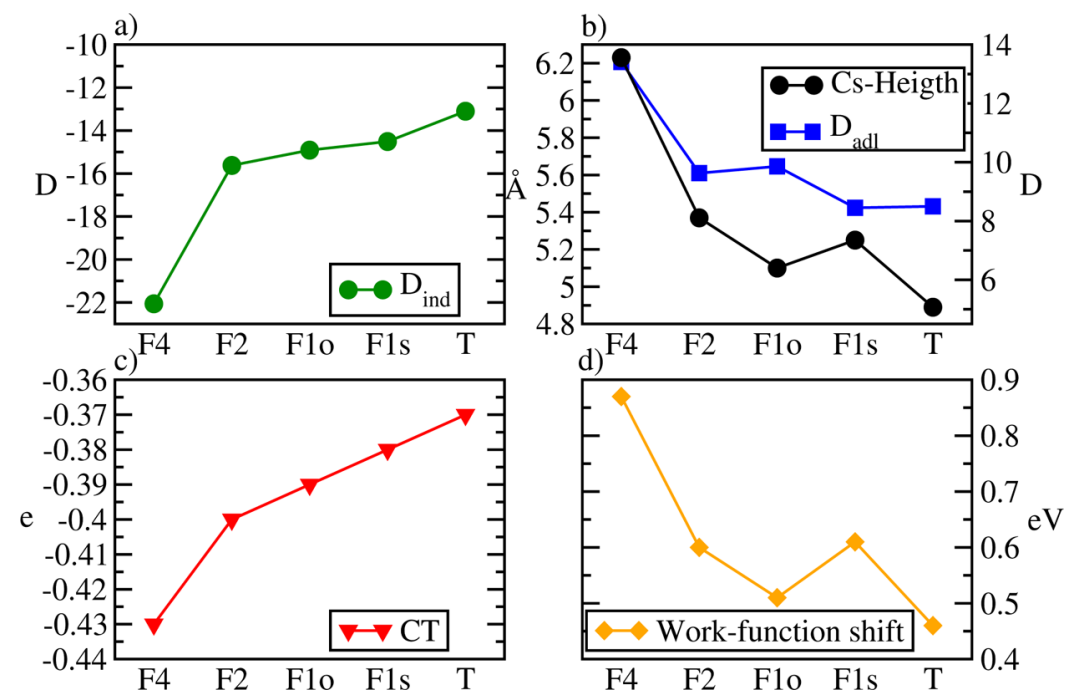

Figure 5. Properties of the Cs-(FD-TCNQ) ${ }_{4}$ complexes on $\mathrm{Ag}(100)$ as a function of the level of fluorination. $F D=F 4$, F2, and F1 report the number of fluorines per molecule. T stands for unsubstituted TCNQ. F1o and F1s are two alternative Cs-(F1-TCNQ) configurations; in one case $\mathrm{F}$ is substituted on the same side where the $\mathrm{Cs}-\mathrm{N}$ bond is ( $\mathrm{F} 1 \mathrm{~s}$ ) and in the other on the opposite side (F1o) (see also the SI). (a) Induced dipole $D_{\text {ind. }}$ (b) Intrinsic dipole $D_{\text {adl }}$ (blue) and Cs height on the substrate (black). The fluorines in F1o and F1s have a different z-coordinate, and this affects both the Cs height and the $D_{\text {adl }}$, see main text. (c) Substrate-adlayer charge transfer per FD-TCNQ molecule. (d) Work-function shift, proportional to the total dipole $D_{\text {ind }}+D_{\text {adlı }}$ clearly correlated to the level of fluorination.

raising the alkali cation position, thus screening the electrostatic repulsion between negatively charged complexes. Nonetheless their usage will increase the work-function shift, as the total interfacial dipole $D_{\text {ind }}+D_{\text {adl }}$ becomes systematically more negative as the adlayer's molecular affinity increases. For the case of a Cs link atom, we predict a $0.87 \mathrm{eV}$ upper limit for the work-function shift, but we emphasize that for lighter alkali metals this limit could further increase (consistent with a lower $D_{\text {adl }}$ ). This significantly enlarges the energy range within which electrostatic tunability could be achieved. Also, we envisage that various levels of molecular fluorination, as well as of different alkali atom mixtures, could be realized within any individual experiment and that further combining these techniques could provide an unusually "robust" parameter domain for experimental work aimed at precise work-function tuning.

\section{CONCLUSIONS}

Alkali metals and TCNQ molecules coadsorbed on a $\mathrm{Ag}(100)$ surface can form extended chiral assemblies of windmill metal-organic complexes, experimentally observed in the case of Cs.

In this work we have investigated via density functional theory the properties of structures containing other alkali atoms ( $\mathrm{Li}, \mathrm{Na}, \mathrm{K})$ for which experiments are not yet available. This led us to identify the specific superstructure observed in Cs as the most probable arrangement even for lighter alkali metals. We find that adsorbing different alkali metals has sizable effects on the adlayer's highly nonplanar structure, while in all cases the TCNQ molecules acquire charge from the $\mathrm{Ag}(100)$ substrate. This affects the interfacial dipoles, ultimately shifting the work-function in a predictable, alkali-dependent fashion, in turn suggesting that mixtures of alkali as metal centers could allow a continuous tuning of the work-function within a fairly large energy interval. The interval could be significantly further increased by considering partially fluorinated TCNQ derivatives, bringing it to an upper limit, which we estimated as $\sim 1 \mathrm{eV}$.

Importantly, such a precise level alignment control would be achieved within a single, self-assembled monolayer made of regular structures, which assemble in large domains and are stable even at room temperature. This route to work-function tuning differs from previous approaches based, for example, on varying the adsorbate coverage or using thick insulating layers (often with a disordered structure) at the MO interface. This layer, composed of oppositely charged donors and acceptors, is structurally more stable against disruptive electrostatic repulsion effects than adlayers of molecules having all the same electronic character. Furthermore, the monolayer is not affected by the depolarization effects, yielding work function saturation once the repelling dipoles become unstable above a certain coverage, as is typical of approaches where the deposition time is used to control the work function at submonolayer coverage. Thanks to its large size, regularity, and extremely reduced thickness, the layer would provide a uniform contact and facilitate transport processes of electrons across the interface. The flexible nature of its bonding structure determines an improved steric performance, as observed for $\mathrm{Cs}_{-} \mathrm{TCNQ}_{4}$ on $\mathrm{Ag}(100)$, possibly leading to large supramolecular assembly domains also on different substrates of choice in specific applications. We speculate that the large work-function energy range and continuous 
tunability pointed out in this work could be appealing also from a manufacturing point of view, where MO interfaces incorporating alkali-linked organic monolayers could be fabricated by direct evaporation through the self-assembly route. The electrostatic stability at room temperature of such partially charged, organic salt monolayers and their combinatorial variety in terms of metal centers and molecular substituents, with the full freedom of using more than one alkali and fluorinated species concurrently, would seem to make this approach particularly promising for foreseeable experimental work aimed at precise work-function control.

\section{METHODS}

Calculations were performed with the planewave-pseudopotential package Quantum-ESPRESSO, ${ }^{32}$ using Ultrasoft pseudopotentials ${ }^{33}$ with a wave function (charge) energy cutoff of $408 \mathrm{eV}(4080 \mathrm{eV})$ and a GGA-PBE ${ }^{34}$ exchange-correlation functional. Brillouin-zone sampling included the $\mathbf{k}=\Gamma$ point only. The adsorption was carried out only on one side of the slab, avoiding spurious dipole effects by means of the technique described in ref $35 . \mathrm{Ag}(100)$ surfaces were modeled with three layers, allowing a vacuum (adlayer-surface distance) of $\sim 10 \AA$. Forces were relaxed up to $0.26 \mathrm{eV} / \AA \AA$. In the presence of the surface, only forces acting on the top layer and the adlayer atoms were relaxed. Charge transfers are calculated with a variation of the method described in ref 36; see also the SI.

Conflict of Interest: The authors declare no competing financial interest.

Acknowledgment. The authors acknowledge support by the EPSRC Grant EP/G044864/1. The experimental image in Figure $1 \mathrm{~b}$ is courtesy of N. Abdurakhmanova and K. Kern. The calculations were performed at the HPC facility of the School of Natural and Mathematical Sciences, King's College London.

Supporting Information Available: Details about the gas-phase calculation; the instability of the $(6,2,-2,6)$ superstructure; the structures for the complexes including fluorinated derivatives; the calculation of the charge transfer and induced dipole. This material is available free of charge via the Internet at http://pubs.acs.org.

\section{REFERENCES AND NOTES}

1. Braun, S.; Salaneck, W. R.; Fahlman, M. Energy-Level Alignment at Organic/Metal and Organic/Organic Interfaces. Adv. Mater. 2009, 21, 1450-1472.

2. Vázquez, H.; Oszwaldowski, R.; Pou, P.; Ortega, J.; Pérez, R.; Flores, F.; Kahn, A. Dipole Formation at Metal/PTCDA Interfaces: Role of the Charge Neutrality Level. Europhys. Lett. 2004, 65, 802.

3. Tang, J. X.; Lee, C. S.; Lee, S. T. Carrier Injection Barrier at Metal-Organic Contacts: Role of Electronegativity. Proceedings of the International Symposium on SuperFunctionality of Organic Devices, IPAP Conference Series 6; Institute of Pure and Applied Physics (IPAP): Japan, 2005, pp 6-20.

4. Koch, N. Energy Levels at Interfaces between Metals and Conjugated Organic Molecules. J. Phys.: Condens. Matter 2008, 20, 184008

5. Gao, W. Y.; Kahn, A. Controlled P-Doping of Zinc Phthalocyanine by Coevaporation with Tetrafluorotetracyanoquinodimethane: A Direct and Inverse Photoemission Study. Appl. Phys. Lett. 2001, 79, 4040.

6. Gao, W. Y.; Kahn, A. Controlled P-Doping of the HoleTransport Molecular Material N,N'-Diphenyl-N, $\mathrm{N}^{\prime}$-Bis(1Naphthyl)-1,1'-Biphenyl-4,4'-Diamine with Tetrafluorotetracyanoquinodimethane. J. Appl. Phys. 2003, 94, 359.

7. Gao, Y. Surface Analytical Studies of Interfaces in Organic Semiconductor Devices. Mater. Sci. Eng., R 2010, 68, 39-87.

8. Liu, X.; Zhan, Y.; Braun, S.; Li, F.; Fahlman, M. Interfacial Electronic Properties of Pentacene Tuned by a Molecular Monolayer of C60. Phys. Rev. B 2009, 80, 115401.

9. Wang, Y.Z.; Qi, D.-C.; Chen, S.; Mao, H.-Y.; Wee, A. T. S.; Gao, X.$Y$. Tuning the Electron Injection Barrier between $\mathrm{Co}$ and $\mathrm{C}_{60}$ Using Alq 3 Buffer Layer. J. Appl. Phys. 2010, 108, 103719.
10. Koch, N.; Duhm, S.; Rabe, J. P.; Rentenberger, S.; Johnson, R. L.; Klankermayer, J.; Schreiber, F. Tuning the Hole Injection Barrier Height at Organic/Metal Interfaces with (Sub-) Monolayers of Electron Acceptor Molecules. Appl. Phys. Lett. 2005, 87, 101905.

11. Greiner, M. T.; Helander, M. G.; Tang, W.-M.; Wang, Z.-B.; Qiu, J.; Lu, Z.-H. Universal Energy-Level Alignment of Molecules on Metal Oxides. Nat. Mater. 2012, 11, 76-81.

12. $\mathrm{Wu}, \mathrm{H} . ;$ Huang, F.; Peng, J.; Cao, Y. High-Efficiency Electron Injection Cathode of Au for Polymer Light-Emitting Devices. Org. Electron. 2005, 6, 118-128.

13. Han, T.-H.; Lee, Y.; Choi, M.-R.; Woo, W.-S.; Bae, S.-H.; Hong, B. H.; Ahn, J.-H.; Lee, T.-W. Extremely Efficient Flexible Organic Light-Emitting Diodes with Modified Graphene Anode. Nat. Photonics 2012, 6, 105-110.

14. Zhou, Y.; Fuentes-Hernandez, C.; Shim, J.; Meyer, J.; Giordano, A. J.; Li, H.; Winget, P.; Papadopulos, T.; Cheun, H.; Kim, J.; et al. A Universal Method to Produce Low-Work Function Electrodes for Organic Electronics. Science 2012, $336,327-332$

15. Helander, M. G.; Wang, Z. B.; Qiu, J.; Greiner, M. T.; Puzzo, D. P.; Liu, Z. W.; Lu, Z. H. Chlorinated Indium Tin Oxide Electrodes with High Work Function for Organic Device Compatibility. Science 2011, 332, 944-947.

16. Hwang, J. O.; Park, J. S.; Choi, D. S.; Kim, J. Y.; Lee, S. H.; Lee, K. E.; Kim, Y.-H.; Song, M. S.; Yoo, S.; Kim, S. O. WorkfunctionTunable, N-Doped Reduced Graphene Transparent Electrodes for High-Performance Polymer Light-Emitting Diodes. ACS Nano 2012, 6, 159-167.

17. Asadi, K.; Gholamrezaie, F.; Smits, E. C. P.; Blom, P. W. M.; De Boer, B. Manipulation of Charge Carrier Injection into Organic Field-Effect Transistors by Self-Assembled Monolayers of Alkanethiols. J. Mater. Chem. 2007, 17, 1947.

18. Bae, G.; Jung, H.; Park, N.; Park, J.; Hong, S.; Park, W. Effect of Charge-Transfer Complex on the Energy Level Alignment between Graphene and Organic Molecules. Appl. Phys. Lett. 2012, 100, 183102.

19. Hanson, E. L.; Guo, J.; Koch, N.; Schwart, J.; Bernasek, S. L. Advanced Surface Modification of Indium Tin Oxide for Improved Charge Injection in Organic Devices. J. Am. Chem. Soc. 2005, 127, 10058-10062.

20. Barth, J. V.; Costantini, G.; Kern, K. Engineering Atomic and Molecular Nanostructures at Surfaces. Nature 2005, 437, 671-679.

21. Ruben, M.; Payer, D.; Landa, A.; Comisso, A.; Gattinoni, C.; Lin, N.; Collin, J.-P.; Sauvage, J.-P.; De Vita, A.; Kern, K. 2D Supramolecular Assemblies of Benzene-1,3,5-TriylTribenzoic Acid: Temperature-Induced Phase Transformations and Hierarchical Organization with Macrocyclic Molecules. J. Am. Chem. Soc. 2006, 128, 15644-15651.

22. Tomba, G.; Stengel, M.; Schneider, W.-D.; Baldereschi, A.; De Vita, A. Supramolecular Self Assembly Driven by Electrostatic Repulsion: The 1D Aggregation of Rubrene Pentagons on Au(111). ACS Nano 2010, 4, 7545-7551.

23. Abdurakhmanova, N.; Floris, A.; Tseng, T. C.; Comisso, A.; Stepanow, S.; De Vita, A.; Kern, K. Stereoselectivity and Electrostatics in Charge-Transfer Mn- and Cs-TCNQ Networks on $\mathrm{Ag}(100)$. Nat. Commun. 2012, 3, 940.

24. Kanai, K.; Akaiake, K.; Koyasu, K.; Sakai, K.; Nishi, T.; Kamizuru, Y.; Nishi, T.; Ouchi, Y.; Seki, K. Determination of Electron Affinity of Electron Accepting Molecules. Appl. Phys. A: Mater. Sci. Process. 2009, 95, 309-313. 
25. Tseng, T. C.; Urban, C.; Wang, Y.; Otero, R.; Tait, S. L.; Alcamí, M.; Ecija, D.; Trelka, M.; Gallego, J. M.; et al. Charge-TransferInduced Structural Rearrangements at Both Sides of Organic/Metal Interfaces. Nat. Chem. 2010, 2, 374-379.

26. Wackerlin, C.; lacovita, C.; Chylarecka, D.; Fesser, P.; Jung, T. A.; Ballav, N. Assembly of 2D lonic Layers by Reaction of Alkali Halides with the Organic Electrophile 7,7,8,8,Tetracyano-P-Quinodimethane (TCNQ). Chem. Commun. 2011, 47, 9146-9148.

27. Database of lonic Radii http://abulafia.mt.ic.ac.uk/shannon/ ptable.php.

28. Rana, O.; Srivastava, R.; Chauhan, G.; Zulfequar, M.; Husain, M.; Srivastava, P. C.; Kamalasanan, M. N. Modification of Metal-Organic Interface Using F4-TCNQ for Enhanced Hole Injection Properties in Optoelectronic Devices. Status Solidi A 2012, 1-7.

29. Romaner, L.; Heimel, G.; Bredas, J.-L.; Gerlach, A.; Schreiber, F.; Johnson, R. L.; Zegenhagen, J.; Duhm, S.; Koch, N.; Zojer, E. Impact of Bidirectional Charge Transfer and Molecular Distortions on the Electronic Structure of a Metal-Organic Interface. Phys. Rev. Lett. 2007, 99, 256801.

30. Koch, N.; Duhm, S.; Rabe, J. P.; Vollmer, A.; Johnson, R. L. Optimised Hole Injection with Strong Electron Acceptor at Organic-Metal Interfaces. Phys. Rev. Lett. 2005, 95, 237601.

31. Rana, O.; Srivastava, R.; Chauhan, G.; Zulfequar, M.; Husain, M.; Srivastava, P. C.; Kamalasanan, M. N. Modification of Metal-Organic Interface Using $\mathrm{F}_{4}$-TCNQ for Enhanced Hole Injection Properties in Optoelectronic Devices. Phys. Status Solidi A 2012, 209, 2539-2545.

32. Gannozzi, P.; Baroni, S.; Bonini, N.; Calandra, M.; Car, R.; Cavazzoni, C.; Ceresoli, D.; Chiarotti, G. L.; Cococcioni, M.; Dabo, l.; et al. www.quantum-espresso.org.

33. Vanderbilt, D. Soft Self-Consistent Pseudopotentials in a Generalized Eigenvalue Formalism. Phys. Rev. B 1990, 41, 7892-7895.

34. Perdew, J. P.; Burke, K.; Ernzerhof, M. Generalized Gradient Approximation Made Simple. Phys. Rev. Lett. 1996, 77, 3865-3868.

35. Bengtsson, L. Dipole Correction for Surface Supercell Calculations. Phys. Rev. B 1999, 59, 12301.

36. Payer, D.; Comisso, A.; Dmitriev, A.; Strunskus, T.; Lin, N.; Wöll, C.; Devita, A.; Barth, J. V.; Kern, K. Ionic Hydrogen Bonds Controlling Two-Dimensional Supramolecular Systems at a Metal Surface. Chem.-Eur. J. 2007, 14, 3900.

37. Leung, T. C.; Kao, C. L.; Su, W. S.; Feng, Y. J.; Chan, C. T. Relationship between Surface Dipole, Work Function and Charge Transfer: Some Exceptions to an Established Rule. Phys. Rev. B 2003, 68, 195408. 